\title{
TECHNISCHE VERFAHREN ZUR SCHADENSANALYSE AN HISTORISCHEN BAUTEN UND DENKMÄLERN
}

\begin{abstract}
Der Beitrag stellt das Methodenspektrum der zerstörungsfreien Prüfverfahren vor, das für die gebaute Infrastruktur einen hohen Sicherheitsstandard gewährleistet. Auch Bauwerke in historischen Gärten können von der Kenntnis dieser Verfahren profitieren. Es werden Regelwerke und Funktion gebräuchlicher zerstörungsfreier Prüfverfahren beschrieben, sodass ein für die jeweilige Fragestellung geeignetes Verfahren ausgewählt werden kann. Die Methoden erlauben ein vorausschauendes Erhaltungsmanagement; das bedeutet, dass Gegenmaßnahmen bereits vor Eintritt von offensichtlichen Schadensbildern getroffen werden können.

This article presents the spectrum of non-destructive test methods that guarantee a high level of security for built infrastructure. Buildings in historic gardens can likewise benefit from an understanding of these methods. The rules and methodology of common non-destructive test methods are described. This helps with the selection of a suitable method for the respective issue. The methods permit forward-looking conservation management. This means that counter-measures can be taken already prior to the appearance of clear evidence of damage.
\end{abstract}

\section{Messen, messen, messen}

Für Galileo Galilei bestand der Kern aller Wissenschaft darin, das zu messen, was messbar ist, und das, was noch nicht messbar ist, messbar zu machen. Bis heute hat sich eine zunehmende Vielfalt an Methoden entwickelt, verschiedenste Parameter auf unterschiedliche Weise bestimmen zu können.

Im Text werden Maskulinum und Femininum verwendet, wenn es um Personen geht. Gemeint sind grundsätzlich alle Menschen, gleich welcher Geschlechtsidentität sie sich zugehörig fühlen. 
Zerstörungsfreie Prüfverfahren und Baukonstruktionen bilden eine Einheit, die einen hohen Sicherheitsstandard der gebauten Infrastruktur gewährleistet.

Bauwerke in historischen Gärten sind Repräsentanten der gebauten Infrastruktur und können vom Kenntnisstand der zerstörungsfreien Prüfung erheblich profitieren. Die folgenden Ausführungen nennen die wichtigsten Regelwerke, erklären die Funktion gebräuchlicher zerstörungsfreier Prüfverfahren (ZfP) und helfen, am besten geeignete Messverfahren $\mathrm{zu}$ finden.

Die zerstörungsfreien Prüfverfahren sind im Beitrag gegliedert nach geringem (G1 bis 8), erhöhtem (M1 bis 11) und hohem technischen Aufwand (H1 bis 10). Die ebenfalls aufgeführten labortechnischen Prüfungen für physikalische (P1 bis 8) und für chemische Kenngrößen (C1 bis 11) liefern, im Gegensatz zu qualitativen Überprüfungen am Bauwerk, quantitative Messergebnisse anhand von Prüfvorschriften.

Einige Beispiele aus den von der Arbeitsgruppe untersuchten Gärten veranschaulichen Auswahl und Einsatz der Prüf- und Messverfahren.

\section{Einsatz von Bauwerksdiagnostik für ein vorausschauendes Erhaltungsmanagement}

Die klassischen Baustoffuntersuchungen stützen sich, im Gegensatz zu den ZfP-Verfahren, auf Norm, Vorschrift, Merkblatt oder Fachliteratur. Der Baustoff und die Bauart bestimmen die Prüfaufgabe. Der mit dem Bauwerk vertraute Ingenieur verfügt über Kerninformationen, der Verfahrensspezialist über Detailwissen. Für die Strategie zur Lösung einer Prüfaufgabe sind folgende Fragen zu beantworten (Hillemeier/Taffe 2012):

\section{Was ist zu messen?}

Die Prüfaufgaben, z.B. die Betondeckung und die Karbonatisierungstiefe, legen beim Beton die Messgröße mit ihrem Zielwert fest. Stein, Holz und Stahl besitzen eigene Messund Zielgrößen.

Bei nichtmetallischen, anorganischen Stoffen wie Stein und Beton stellen die Zusammensetzung und das Porensystem die jeweiligen Zielgrößen dar. Schwinden, Quellen und Auflösen infolge von Sorption und Kapillaraktivität können zerstörend wirken. Anzeichen einer Zerstörung sind Änderungen von Farbe, Festigkeit und Steifigkeit. Noch verborgene Anzeichen erkennt man mit Antwortspektren aus mechanischen Wellenimpulsen wie Ultraschall oder Impakt-Echo oder elektromagnetischen Impulsen wie Radar oder Impulsthermographie. Probenanalytisch schlüsselt man die Stoffe mit der Atomabsorptionsspektroskopie (AAS),»Laser Induced Breakdown Spectroscopy« (LIBS), Röntgenfluoreszenzanalyse (RFA) und Energiedispersive Röntgenspektroskopie (EDX) auf. 


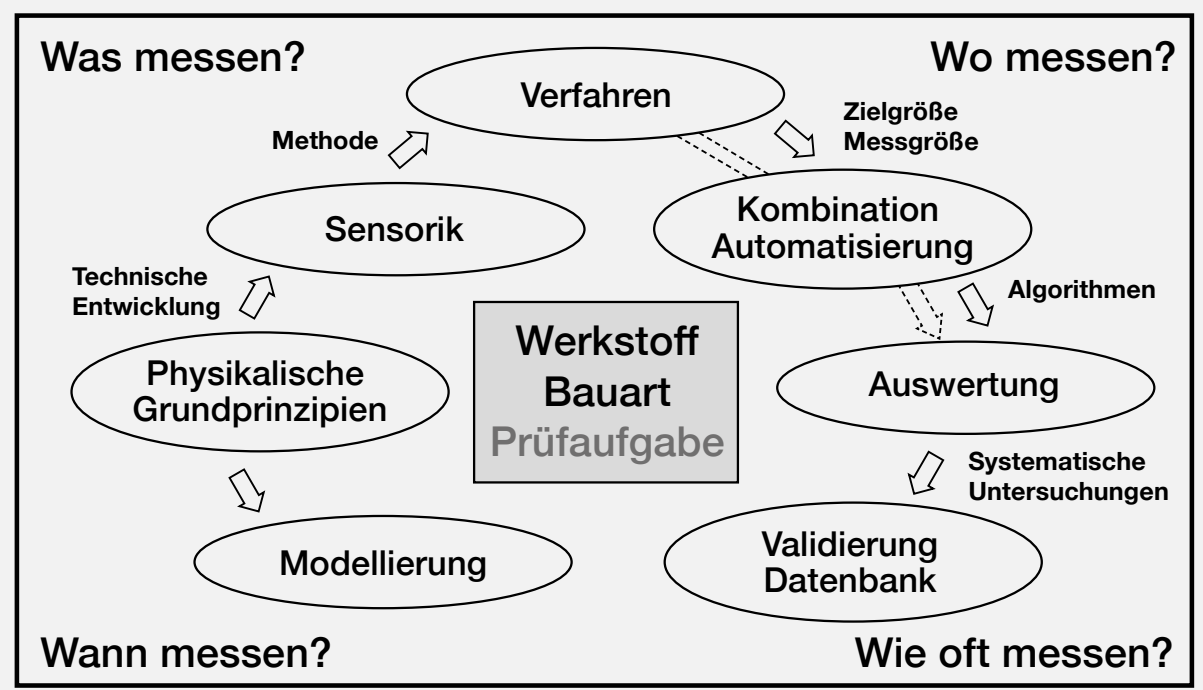

\section{Prüfaufgabe $\Rightarrow$ Strategie $\Rightarrow$ Zuverlässige Prüfaussage}

1 Multidisziplinarität der Bauwerkdiagnose und die Bedeutung einer Strategie zur Lösung von Prüfaufgaben (Bauministerkonferenz - Konferenz für den Städtebau, Bau- und Wohnungswesen zuständigen Senatoren der Länder [ARGEBAU] 2006).

\section{Wo soll gemessen werden?}

Hierzu bedarf es sowohl eines Detailwissens zu Statik und Mechanik als auch einer Kenntnis der Schwachstellen der Konstruktion sowie zu Schadensmechanismen des Baustoffs, wie z.B. bei Stahlbeton eine tausalzinduzierte Korrosion oder Alkali-Kieselsäure-Reaktion (AKR). Weil dieses Wissen bei Verfahrensspezialisten nicht vorausgesetzt werden kann, sollten Messstellen zusammen mit Personen festgelegt werden, die die Konstruktion und ihre Schwachstellen kennen. Bei Brückenbauwerken sind das die Bauwerksprüfer, bei Bauten in historischen Gärten die Denkmalämter. (Abb.1.)

Die folgenden 10 Regeln für eine Bestandsaufnahme an einem Bauwerk dienen der vorausschauenden Planung:

1. Inspektion und Analyse durch einen sachkundigen Bauingenieur hinsichtlich Statik, Konstruktion, Werkstoff, Brandschutz und Bauphysik.

2. Beachtung der technischen Baubestimmungen und der Grundsätze des Bestandsschutzes.

3. Der Bestandsschutz räumt das Recht ein, bauliche Anlagen abweichend von geltendem Recht instand zu halten.

4. Instandhalten, Ändern und Rückbauen baulicher Anlagen nach den eingeführten Technischen Baubestimmungen (ETB). 
5. Der Verfahrensspezialist und der Bauingenieur arbeiten disziplinübergreifend zusammen.

6. Der professionelle Bauwerksprüfer kennt die Schwachstellen der Konstruktion und ist ggf. als dritter Spezialist hinzuzuziehen.

7. Die Verlässlichkeit eines Prüfverfahrens und die Zuverlässigkeit einer Prüfaussage sind vor der Prüfung zu erklären und zu dokumentieren.

8. Der Verfahrensspezialist kennt und erklärt die Grenzen des Prüfverfahrens in Abhängigkeit von den Randbedingungen des Messeinsatzes.

9. Vor einer Bauwerksprüfung wird abgestimmtes Handeln festgelegt: Wie handelt man bei einem positiven Messergebnis, wie bei einem negativen und wie bei einer unsicheren Aussage? Bleibt eine dieser Möglichkeiten offen, hilft eine Prüfung nicht weiter.

10. Planung, Durchführung, Auswertung und Bewertung einer Bestandsaufnahme sind nach den Prinzipien eines Qualitätsmanagementsystems mit Aufbauorganisation, Ablauforganisation und Dokumentation auszuführen.

\section{Die wichtigsten Regelwerke zur Bauwerksprüfung}

\subsection{DIN 1076}

Die Bauwerksprüfung nach DIN 1076 unterscheidet in Hauptprüfungen (alle 6 Jahre), Einfache Prüfungen (3 Jahre nach einer Hauptprüfung) und Prüfungen aus besonderem Anlass (Feuer oder Anprall) (DIN 1076). Bauwerksprüfungen sind von einem »Sachkundigen Ingenieur «, Maßnahmen zur Bauwerküberwachung von einer »Sachkundigen Person « vorzunehmen.

\subsection{RÜV}

Die Richtlinie für die Überwachung der Verkehrssicherheit von baulichen Anlagen des Bundes (RÜV) regelt »Verantwortlichkeiten und Zuständigkeiten bei der Überwachung der Standund Verkehrssicherheit « und teilt auf der Grundlage von Bauwerksakten Bauwerke in die Klassen vorrangig (Klasse 1) und nachrangig (Klasse 2) ein (RÜV). Die Festlegung trifft die zuständige Bauverwaltung.

\subsection{OSA Leitfaden zur Objektbezogenen Schadensanalyse}

Der Leitfaden Objektbezogene Schadensanalyse (OSA) der Bundesanstalt für Straßenwesen (BASt) beschreibt die Vorgehensweise bei einem Schaden, der über die Bauwerksprüfung 
nach DIN 1076 hinausgehende Untersuchungen erfordert (Bundesministerium für Verkehr, Bau und Stadtentwicklung 2017).

Er verweist auf die Verfahrensmerkblätter der DGZfP (Deutsche Gesellschaft für zerstörungsfreie Prüfung, vgl. Abschnitt 8.1).

\subsection{VDI-Richtlinie 6200}

Die VDI-Richtlinie Standsicherheit von Bauwerken - Regelmäßige Überprüfung (VDI 6200) wurde auf Grundlage der Hinweise der Bauministerkonferenz aus dem Jahr 2006 (nach dem Einsturz der Eissporthalle in Bad Reichenhall) erstellt (VDI-Gesellschaft Bauen und Gebäudetechnik, Fachbereich Bautechnik 2010). Sie beschreibt eine strukturierte Vorgehensweise von der Einstufung in Schadensfolgeklassen, Robustheitsklassen und Überprüfungsintervalle mit Überprüfungsmethoden und den Überprüfungsstufen: Begehung, Inspektion und Eingehende Überprüfung durch eine Besonders Fachkundige Person.

Als Fachkundige Personen gelten demnach Bauingenieure und Architekten mit mindestens fünf Jahren Erfahrung in der Aufstellung von Standsicherheitsnachweisen oder technischer Bauleitung, die bei Besonders Fachkundigen Personen mindestens zehn Jahre betragen muss. Ingenieurkammern der Länder führen Listen dieser Personen.

\subsection{RL SIB}

Die DAfStb-Richtlinie Schutz und Instandsetzung von Betonbauteilen (RL SIB) des Deutschen Ausschusses für Stahlbeton nennt Verfahren zur Instandsetzung (DAfStb-Richtlinie - Schutz und Instandsetzung von Betonbauteilen 2001-2010).

Diese Auflistung zeigt, dass es bereits eine Reihe von Regelwerken gibt, die für eine Begutachtung von Bauwerken in historischen Gärten herangezogen werden können.

\section{Verfahren zur klassischen Baustoffprüfung}

Visuell werden auf Oberflächen mineralischer Werkstoffe (Beton, Naturstein, Mauerwerk) Ausblühungen, Gelausscheidungen, Abwitterungen, Abplatzungen, Risse, Fehlstellen, Rostabläufer, Schmutzfahnen, Salzanreicherungen, biologischer Bewuchs und Durchfeuchtungen festgestellt.

Die Tabellen 1 bis 3 unterscheiden baustellenpraktische Verfahren zur Messung der Ursachen der vorgenannten Auffälligkeiten nach geringem, erhöhtem und hohem technischen Aufwand.

Im Vorangehenden wurden Messverfahren zusammengestellt, die neben visuellen Kategorien vor allem baustellenpraktische Methoden benennen. Im Folgenden werden einige Verfahren skizziert. 


\begin{tabular}{|c|c|c|c|}
\hline & Untersuchungsziel (G=gering) & Prüfverfahren & Abschnitt \\
\hline G1 & Verfärbungen, Risse, Besonderheiten & Augenschein & [7] \\
\hline G2 & Oberflächenrauigkeit & Sandflächenverfahren & \\
\hline G3 & Feuchtigkeitszustand oberflächennah & Trocknen mit Warmluft & \\
\hline \multirow[t]{3}{*}{ G4 } & $\begin{array}{l}\text { Dichtigkeit einer Bauteiloberfläche. } \\
\text { Wirksamkeit einer Hydrophobierung }\end{array}$ & Wasserspritzflasche & \\
\hline & & Prüfröhrchen nach Karsten & 5.1 \\
\hline & & ISA-Test & 5.1 \\
\hline \multirow[t]{2}{*}{ G5 } & Rissbreiten & Rissbreitenvergleichsmaßstab & $\begin{array}{l}5.7 .1 \\
5.7 .2\end{array}$ \\
\hline & & Risslupe & \\
\hline G6 & Rissbreitenänderung (qualitativ) & Gipsmarke & \\
\hline \multirow[t]{2}{*}{ G7 } & Rissbreitenänderung (quantitativ) Rissmonitoring & Eurogard & \\
\hline & & Scratch-a-track & \\
\hline G8 & Lage der Bewehrung & Magnet & 5.8 \\
\hline
\end{tabular}

Tabelle 1 Prüfverfahren mit geringem technischen Aufwand

\section{Erläuterung relevanter Prüfverfahren}

\subsection{Saugfähigkeit mit dem Prüfröhrchen nach Karsten, ISA-Test}

Genauer als mit der Spritzflasche lässt sich die kapillare Wasseraufnahme (Saugfähigkeit) mit dem Prüfröhrchen nach Karsten bestimmen. International ist das Verfahren verfeinert worden (ISAT = Initial Surface Adsorption Test).

Der Feuchtegehalt lässt sich noch genauer an einer entnommenen Probe ermitteln, die für die Laboruntersuchung sofort nach der Entnahme gewogen und luftdicht verpackt werden muss.

\subsection{Feuchtemessung mit dem CM-Gerät und mit Mikrowellentechnik}

An Ort und Stelle lässt sich die Bauteilfeuchte mit Hilfe der Calciumcarbid-Methode (CM) bestimmen. Eine zerkleinerte und auf $2 \mathrm{~mm}$ abgesiebte Probe reagiert mit ihrer Feuchte in einem Stahlbehälter mit Manometer, in den Calciumcarbid gegeben wird, zu Acetylengas. Eine Tabelle nennt den Feuchtigkeitsgehalt in Abhängigkeit vom Gasdruck. 


\begin{tabular}{|c|c|c|c|}
\hline & Untersuchungsziel ( $M=$ mittel) & Prüfverfahren & Abschnitt \\
\hline \multirow[t]{2}{*}{ M1 } & $\begin{array}{l}\text { Feuchtegehalt konventionell, kapazitiv, } \\
\text { mit Mikrowellen und durch elektrische } \\
\text { Widerstandsmessung }\end{array}$ & Wägung, Trocknung, & \\
\hline & & CM-Gerät, Moist Scan & 5.2 \\
\hline M2 & Gasdurchlässigkeit, Dichtigkeit & Permeabilitätsmessgerät & \\
\hline M3 & Festigkeitsbestimmung an mineralischen Baustoffen & Rückprallhammer (Schmidt) & \\
\hline M4 & Oberflächenzugfestigkeit Haftzugfestigkeit & Haftzugprüfgerät (mobil) & 5.3 \\
\hline M5 & $\begin{array}{l}\text { Druckfestigkeit, E-Modul, Betongefüge, } \\
\text { Chloridprofil, } \\
\text { Alkalikieselgel, Rissart und Risszustand }\end{array}$ & $\begin{array}{l}\text { Kernbohrgerät zur } \\
\text { Bohrkernentnahme }\end{array}$ & \\
\hline M6 & Karbonatisierungstiefe & $\begin{array}{l}\text { Farbumschlagprüfung } \\
\text { mit Phenolphthalein- } \\
\text { Sprühflüssigkeit }\end{array}$ & 5.4 \\
\hline M7 & Chloridgehalt (qualitativ) & $\begin{array}{l}\text { Farbumschlagprüfung } \\
\text { mit Silbernitrat- und } \\
\text { Kaliumchromatlösung }\end{array}$ & 5.5 \\
\hline M8 & Betondeckung, Bewehrungsortung und -verlauf & Überdeckungsmessgeräte & $\begin{array}{l}8.1(\mathrm{~B} 02) \\
5.9 \\
8.1(\mathrm{~B} 03)\end{array}$ \\
\hline M9 & Korrosion der Bewehrung & $\begin{array}{l}\text { Potentialmessung mit der } \\
\text { Kupfer-Kupfersulfat-Elektrode } \\
\left(\mathrm{Cu} / \mathrm{CuSO}_{4}\right) \text { (mobil) }\end{array}$ & \\
\hline \multirow[t]{2}{*}{ M10 } & Rissbreitenänderung & Messuhr & \\
\hline & & Induktiver Wegaufnehmer & \\
\hline M11 & Haftung und Güte von Beschichtungen & Gitterschnittverfahren & [7] \\
\hline
\end{tabular}

Tabelle 2 Prüfverfahren mit erhöhtem technischen Aufwand

Das Moist-Scan Gerät ermöglicht auf der Basis von Mikrowellentechnik mit drei am Scanner befestigten Sonden, die verschiedene Einwirktiefen haben, eine Aussage zur Tiefenschichtung im Wand- oder Bodenaufbau.

\subsection{Oberflächenzugfestigkeit und Haftzugfestigkeit}

Für Oberflächenschutzschichten werden die Oberflächenzugfestigkeit des Betonuntergrundes und die Haftzugfestigkeit des aufgebrachten Beschichtungsstoffs bestimmt. Eine mittlere Haftzugfestigkeit von $1,5 \mathrm{~N} / \mathrm{mm}^{2}$ gilt als Schwellenwert. Der Wert 1,5 N/mm 2 ergibt sich rechnerisch für einen Beton vergleichbar der Festigkeitsklasse C 20/25. Wird 


\begin{tabular}{|c|c|c|c|}
\hline & Untersuchungsziel (H=hoch) & Prüfverfahren & Abschnitt \\
\hline $\mathrm{H} 1$ & $\begin{array}{l}\text { Einsicht in Hohlräume, Dokumentation von } \\
\text { Schäden, Rissen und Korrosion im Inneren von } \\
\text { Bauteilen }\end{array}$ & $\begin{array}{l}\text { Endoskop (flexibel), } \\
\text { Boreskop (starr) }\end{array}$ & 5.10 \\
\hline $\mathrm{H} 2$ & $\begin{array}{l}\text { Spannstahlortung, Dickenmessung von Bauteilen, } \\
\text { Ortung von Inhomogenitäten }\end{array}$ & Radar & 5.11 \\
\hline \multirow[t]{2}{*}{$\mathrm{H} 3$} & $\begin{array}{l}\text { Ortung von Hohlstellen, Rissen und } \\
\text { Gefügeauflockerungen }\end{array}$ & Ultraschall & $\begin{array}{l}8.1(\mathrm{~B} 04) \\
5.13\end{array}$ \\
\hline & & Impakt-Echo & 5.12 \\
\hline $\mathrm{H} 4$ & Ortung von Inhomogenitäten, Feuchteverteilung & Infrarot-Thermographie & 5.14 \\
\hline H5 & $\begin{array}{l}\text { Ortung von Hohlstellen, Rissen und Einbauteilen, } \\
\text { Beurteilung des Verpresszustands von Hüllrohren }\end{array}$ & Röntgen-, Gammastrahlgerät & 5.15 \\
\hline H6 & Bauteil- bzw. Bauwerkverschiebungen & $\begin{array}{l}\text { Geodätische Messverfahren, } \\
\text { Bau-Laser }\end{array}$ & 6.3 \\
\hline $\mathrm{H7}$ & $\begin{array}{l}\text { Schwingungsverhalten eines Bauteils oder } \\
\text { Bauwerks }\end{array}$ & $\begin{array}{l}\text { Weg- und } \\
\text { Beschleunigungsaufnehmer }\end{array}$ & \\
\hline $\mathrm{H} 8$ & $\begin{array}{l}\text { Dehnungen von jungem und erhärtetem Beton, } \\
\text { Rissbewegungen }\end{array}$ & Glasfasersensoren & 6.2 \\
\hline $\mathrm{H} 9$ & Oberflächenrissprüfung & $\begin{array}{l}\text { Magnetpulverprüfung } \\
\text { Farbeindringverfahren }\end{array}$ & \\
\hline $\mathrm{H} 10$ & $\begin{array}{l}\text { Schichtdickenmessung der Korrosionsschutz- } \\
\text { beschichtung bzw. metallischer Überzüge }\end{array}$ & Wirbelstrom & \\
\hline
\end{tabular}

Tabelle 3 Prüfverfahren mit hohem technischen Aufwand

dieser Wert unterschritten, muss der Tragwerkplaner entscheiden, ob der Beton für die geplante Instandsetzung noch geeignet oder zu wenig haftfest ist.

\subsection{Messen der Karbonatisierungstiefe}

Um herauszufinden, inwieweit im Beton eingebetteter Stahl vor Korrosion geschützt ist, misst man die Karbonatisierungstiefe. Die Karbonatisierungstiefe eines Betons erkennt man anhand eines Farbumschlags. Eine Phenolphthalein-Lösung schlägt bei einem pH-Wert oberhalb von etwa 8,0 von farblos zu rot/violett um. Bei diesem Farbumschlag ist der Beton alkalisch, nicht karbonatisiert und schützt eingebetteten Stahl vor Korrosion. Zur Messung der Karbonatisierungstiefe wird eine frische Bruchfläche mit der Indikatorflüssigkeit besprüht. Trockener Beton muss vor dem Aufsprühen angefeuchtet werden. 


\subsection{Bestimmung des Chloridgehalts durch Tausalze}

Eine Lösung aus Silbernitrat und Kaliumchromat färbt chloridfreien Beton braun und chloridhaltigen Beton gelb. Der Farbumschlag liegt bei 0,4 M.-\% Chlorid bezogen auf den Zement. Die Werte 0,4 M.-\% für Stahlbeton und 0,2 M.-\% für Spannbeton gelten nach der EN 206-1 noch als ungefährlich.

\subsection{LIBS zur Messung des Chloridgehalts und der Elementeverteilung}

Die »Laser Induced Breakdown Spectroscopy« (LIBS) bietet die Möglichkeit, direkt auf der Oberfläche von Baustoffen zweidimensional die Elementeverteilung zu erfassen. Die Vorteile des Verfahrens liegen in der einfachen Probenvorbereitung, der direkten Messung auf der Oberfläche des Festkörpers und der Schnelligkeit der Messung.

\subsection{Untersuchung von Rissen}

Risse in Stahlbeton sind nicht notwendigerweise ein Schaden. Die hierfür gültige Richtlinie DIN EN 1992-1·1 erfasst nur Risse mit einer Rissbreite größer 0.2 mm. Risse können aber ein Hinweis auf statische Probleme sein oder einen Schadensprozess fördern, etwa die Depassivierung der Bewehrung durch die beschleunigte Diffusion von Kohlendioxid. Kohlendioxid wandelt das den Stahl schützende Kalziumhydroxid im Beton in neutralen Kalkstein um. Der dadurch ungeschützte Stahl rostet dann bei Feuchtezutritt. Deshalb ist die Bestimmung der Risstiefe und des Rissverlaufs für die Dauerhaftigkeitsabschätzung eines Bauwerks von Interesse (Tabelle 4).

\subsubsection{Rissbreitenvergleichsmaßstab}

Bei Rissen werden Zustand, Risslänge, Risstiefe und Rissbreite bestimmt. Ihre Breite lässt sich mit einem Rissbreitenvergleichsmaßstab messen.

\begin{tabular}{l|l|l}
\hline Rissart & Ort des Auftretens & Ursache \\
\hline Setzrisse & $\begin{array}{l}\text { Längsrisse entlang und über der oben } \\
\text { liegenden Bewehrung, am oberen } \\
\text { Ende von Stützen, an Stellen von } \\
\text { Dickenänderungen }\end{array}$ & $\begin{array}{l}\text { frühzeitiges Austrocknen und/oder } \\
\text { starkes Absetzen des Betons }\end{array}$ \\
\hline Frühschwindrisse & $\begin{array}{l}\text { Bauteile mit ausgedehnter waagerechter } \\
\text { Oberfläche, z.B. Deckenplatten, diagonal } \\
\text { verlaufende Risse oder Netzrisse }\end{array}$ & $\begin{array}{l}\text { schnelles oder frühzeitiges Austrocknen, } \\
\text { mehlkornreiche Betone }\end{array}$ \\
\hline
\end{tabular}

Tabelle 4.1 Erscheinungsformen, Ort des Auftretens und Ursachen von Rissen 


\begin{tabular}{l|l|l}
\hline Temperaturrisse & bei dünnen Platten und Wänden & klimabedingte Temperaturunterschiede \\
\hline Schwindrisse & bei dünnen Platten und Wänden & $\begin{array}{l}\text { Trocknungsschwinden, schlechte } \\
\text { Nachbehandlung }\end{array}$ \\
\hline Krakelee-Risse & bei geschalten Flächen (Netzrisse) & $\begin{array}{l}\text { nicht saugfähige Schalung, feinteilreiche } \\
\text { Mischungen, } \\
\text { schlechte Nachbehandlung. }\end{array}$ \\
\hline $\begin{array}{l}\text { Risse infolge } \\
\text { Bewehrungs- } \\
\text { korrosion }\end{array}$ & bei Platten (Netzrisse) & $\begin{array}{l}\text { starkes Glätten, Totreiben, feinteilreiche } \\
\text { Mischung }\end{array}$ \\
\hline $\begin{array}{l}\text { Risse als Signale } \\
\text { aus dem } \\
\text { Untergrund }\end{array}$ & $\begin{array}{l}\text { z.B. Bauteile über Pfahlgründungen aus } \\
\text { Holz }\end{array}$ & $\begin{array}{l}\text { unzureichende Dichte und Dicke der } \\
\text { Betondeckung. }\end{array}$ \\
\hline
\end{tabular}

Tabelle 4.2 Erscheinungsformen, Ort des Auftretens und Ursachen von Rissen

\subsubsection{Rissbreitenänderung}

Unter rissüberbrückenden Beschichtungen sollen Risse ruhend sein. Dies lässt sich mit etwa $3 \mathrm{~mm}$ dicken, Zwei-Euro-Stück großen Gipsmarken nachweisen. Eine Temperaturänderung von $10 \mathrm{~K}$ führt bei einem $6 \mathrm{~m}$ langen Bauteil an einem mittigen Einzelriss zu einer Rissbreitenänderung von $0,3 \mathrm{~mm}$. Dehnungen dieser Größenordnung erträgt kein rissüberbrückendes System dauerhaft. Rissbreitenänderungen können mit einem Rissbreitenvergleichsmaßstab ermittelt werden.

Das Bewegungsspiel von Rissen wird mit elektronischen Wegaufnehmern und mechanischen Messuhren gemessen.

\subsection{Bewehrungsortung und Betondeckung}

Mit Neodym-Permanentmagneten lässt sich die Bewehrung auf der Betonoberfläche bis 15 mm Tiefe erfühlen. Haftet ein Magnet am Beton, so ist die Betondeckung unzureichend.

\subsection{Potentialmessung zur Korrosionserkennung}

Die elektrochemische Potentialmessung zeigt die Korrosionsbereitschaft von Bewehrungsstählen. Der Beton muss ionenleitfähig sein und ist ggf. vor der Messung anzufeuchten. Tabelle 5 zeigt die Bewertung der Messung. 


\begin{tabular}{l|l}
\hline Potential U $\left(\mathrm{Cu} / \mathrm{CuSO}_{4}\right)$ & Bewertung nach ASTM C 876-91 \\
\hline$U>-200 \mathrm{mV}$ & Mit über $90 \%$ Wahrscheinlichkeit keine Korrosion. \\
\hline$-350 \mathrm{mV} \leq-200 \mathrm{mV}$ & Keine Aussage über das Korrosionsverhalten. \\
\hline$U<-350 \mathrm{mV}$ & Mit über $90 \%$ Wahrscheinlichkeit Korrosion. \\
\hline
\end{tabular}

Tabelle 5 Grenzwerte und Bewertung einer Stahlkorrosion

\subsection{Endoskopie}

Endoskope blicken in verborgene Hohlräume, wobei der Eingriff in die Bausubstanz gering ist. Nur kleine Bohröffnungen $(\varnothing<15 \mathrm{~mm})$ sind erforderlich. Man unterscheidet zwischen flexiblen Endoskopen und starren, jedoch lichtstärkeren Boreskopen.

\subsection{Radar}

Elektromagnetische Radarwellen werden an Grenzflächen mit unterschiedlichen dielektrischen Eigenschaften reflektiert. Gut detektiert werden metallische Hüllrohre, schlaffe Bewehrung, Hohlstellen und Einbauteile sowie die Dicke von Bauteilen. Ein Hohlraum wird detektiert, wenn sein Durchmesser mindestens so groß ist wie seine Tiefenlage. Mit großen Georadarantennen $(900 \mathrm{MHz})$ können Rückschlüsse auf den Zustand der unter einer Straßenoberfläche liegenden Bettungsschichten gezogen werden.

Der Trend zur schnellen bildgebenden Darstellung von Radarergebnissen wird mit einem Radarkompaktgerät bedienerfreundlich umgesetzt.

Das Radarverfahren detektiert Fäulnis in Bäumen oder Fäulnis- und Insektenschäden an Holzbrücken aus Baumstämmen.

\subsection{Impakt-Echo}

Das Impakt-Echo-Gerät führt kurze mechanische Schläge auf eine Bauteiloberfläche aus. Die »Impakts« regen das Bauteil zu komplexen Schwingungsmoden an. Sensoren nehmen die Signale auf der Oberfläche auf. Im Gegensatz zum Ultraschall ist kein Kopplungsmittel zur Schalleintragung (Vaseline, Öl) erforderlich. Das Verfahren eignet sich gut zur Dickenmessung. Es reagiert zugleich sensitiv auf strukturelle Schädigungen und lässt sich auch zur Ableitung von Materialparametern einsetzen. 


\subsection{Ultraschall}

Ultraschall breitet sich als elastische Welle im Bauteil aus und wird an Schichtgrenzen reflektiert oder an Reflektoren (Bewehrungsstäbe, Hüllrohre, Einbauteile) rückgestreut. Eine Grenzfläche zu Luft bewirkt Totalreflexion. Hinter dieser Grenze können keine Informationen gewonnen werden. Aus der Laufzeit des reflektierten Impulses lässt sich die Entfernung des Reflektors berechnen. Konventionelle Systeme zur Ultraschall-Echo-Prüfung von Betonbauteilen sind in ihrer Eindringtiefe auf etwa einen Meter begrenzt. Zur Prüfung dickerer Objekte dient das LAUS-System (Large Aperture Ultrasound), bestehend aus einzelnen Arrays, die mit bis zu 40 Einzelprüfköpfen als Sender oder Empfänger betrieben werden können.

Für die Ultraschall-Dickenmessung von Betonbauteilen sind Handmessgeräte verfügbar, mit denen einseitige Messungen schnell und einfach durchgeführt werden können.

Auch die Ultraschalltechnik ermöglicht eine bildgebende Darstellung.

\subsection{Infrarot-Thermographie}

Für die Ortung von oberflächennahen Inhomogenitäten findet auch die Infrarot-Thermographie Anwendung. Hohlstellen, Hohllagen von Estrichschichten, Risse und Gegenstände im Bauteil werden durch einen dort gestörten Wärmeabfluss sichtbar gemacht.

\subsection{Röntgen- und Gammastrahlung}

Die Durchstrahlungsmethode bildet auf einer Filmplatte Einbauteile und Hohlräume in hoher Detailtreue ab. Bedingung ist die beiderseitige Zugänglichkeit des zu untersuchenden Bauteils. Die maximal durchstrahlbare Bauteildicke liegt für Beton bei $60 \mathrm{~cm}$. Es sind die Vorkehrungen des Strahlenschutzes zu beachten.

\section{Monitoring}

Monitoringkonzepte setzen analytische Vorüberlegungen voraus. Aus dem Trend der Messergebnisse bewertet man den Zustand eines Bauwerks hinsichtlich Tragfähigkeit, Dauerhaftigkeit und Gebrauchstauglichkeit.

\subsection{Fasersensorik}

Faseroptische Sensoren messen physikalische Größen wie Kraft, Weg und Dehnung, aber auch thermodynamische und chemische Größen wie Temperatur, Feuchtigkeit und pHWert. Faseroptische Sensoren haben den Vorteil, unbeeinflusst von elektromagnetischen 
Feldern zu sein, und das bei guter Korrosionsunempfindlichkeit, Messung über lange Messstrecken sowie geringen Kosten.

\subsection{Weg-, Beschleunigungs- und Kraftaufnehmer}

Weg-, Beschleunigungs- und Kraftaufnehmer registrieren das Schwingungsverhalten eines Bauwerks. Veränderungen der Messergebnisse lassen Rückschlüsse auf Strukturveränderungen und ggf. auf Schäden zu.

\section{Laboruntersuchungen}

Laboruntersuchungen liefern im Gegensatz zu qualitativen Überprüfungen am Bauwerk quantitative Messergebnisse anhand von Prüfvorschriften.

Physikalische Analysegeräte geben Informationen über die Stoffzusammensetzung: über die Elemente mit der Elementanalyse, über den atomaren Aufbau von Molekülen mit der Struktur-Analyse und über Bindungskräfte mit den Molekül-Analyse-Verfahren. Die Emissionsspektralanalyse analysiert Metalle. Eine blankgeschliffene Probe wird durch einen Funken abgefunkt. Dabei verdampft eine geringe Menge des Materials, das im Lichtbogen seine charakteristischen Spektren emittiert.

\subsection{Entnahme von Proben}

Bohrkerne geben sofort Aufschluss über die Kornabstufung einer Gesteinskörnung. Eine Harzinjektion vorab kann einen empfindlichen Risszustand während der Bohrkernentnahme »einfrieren«. Die Probe bleibt bei der Entnahme erhalten und zerbröselt nicht (Tabellen 6,7).

\subsection{Licht- und Rasterelektronen-Mikroskopie}

Schädigungsmechanismen wirken über das Porengefüge. Die Porenform wird mit lichtmikroskopischen Bildanalysen an Dünnschliffen getrockneter Betonproben oder mit rasterelektronen-mikroskopischen Aufnahmen sichtbar gemacht. Die Lichtmikroskopie misst Porengrößen ab $5 \mu \mathrm{m}$.

\subsection{Durchlässigkeitsprüfung}

Der durchströmbare offene Porenanteil wird anhand einer Permeabilitätsmessung über ein Druckgefälle an einer scheibenförmigen Probe in einem speziellen Prüfstand ermittelt. 


\begin{tabular}{l|l|l|l}
\hline & Prüfverfahren & Untersuchungsziel & Abschnitt \\
\hline P1 & Universalprüfmaschine & Zug- / Druckfestigkeit, statischer E-Modul & \\
\hline P2 & Ultraschallprüfung & dynamischer E-Modul & \\
\hline P3 & Längenänderung & Quellen, Schwinden, Treiben & \\
\hline P4 & Frost-Tau-Wechsel-Prüfung & Frostwiderstand & \\
\hline P5 & \multirow{2}{*}{ Wägung, Messung } & $\begin{array}{l}\text { Rohdichte, Reindichte, Trockenrohdichte, } \\
\text { Feuchtegehalt, Gesamtporosität }\end{array}$ & \\
\cline { 3 - 4 } & & Bezugsfeuchtegehalt & \\
\cline { 3 - 4 } & & Kapillare Wasseraufnahme & 7.4 \\
\cline { 3 - 4 } & & Diffusionswiderstand & \\
\hline P6 & \multirow{2}{*}{ Durchlässigkeitsprüfstand } & Gasdurchlässigkeit & \\
\cline { 3 - 4 } & & Wasserdurchlässigkeit & $7.3+7.5$ \\
\hline P7 & \multirow{2}{*}{$\begin{array}{l}\text { Quecksilberdruck-Porosimetrie, } \\
\text { BET-Verfahren }\end{array}$} & $\begin{array}{l}\text { Gesamtporosität, Porenradienverteilung, innere } \\
\text { Oberfläche }\end{array}$ & \\
\hline & Rasterelektronen-Mikroskopie & $\begin{array}{l}\text { Betonzusammensetzung, Baustoffgefüge, Poren, } \\
\text { Abstandsfaktor der Poren }\end{array}$ & \\
\hline
\end{tabular}

Tabelle 6 Labortechnische Prüfverfahren zur Bestimmung physikalisch-mechanischer Eigenschaften

\begin{tabular}{l|l|l|l}
\hline & Prüfverfahren & Untersuchungsziel & Abschnitt \\
\hline C1 & $\begin{array}{l}\text { Elektrometrische } \\
\text { pH-Wert-Bestimmung }\end{array}$ & $\begin{array}{l}\text { Karbonatisierungstiefe, } \\
\text { Karbonatisierungsfortschritt }\end{array}$ & \\
\hline C2 & Nebelkammerlagerung & $\begin{array}{l}\text { Nachweis von Alkalikieselgel bei Vermutung einer } \\
\text { Alkali-Silika-Reaktion (AKR) }\end{array}$ & \\
\hline C3 & NBRI/RILEM-Test & & \\
\hline C4 & $\begin{array}{l}\text { Uranylacetat-Lösung im UV- } \\
\text { Licht }\end{array}$ & & \\
\hline C5 & Röntgenfluoreszenz & Elementverteilungsanalyse & \\
\hline C6 & $\begin{array}{l}\text { Gaschromatographie } \\
\text { Massenspektrometrie }\end{array}$ & Organische Verbindungen (baustoffschädigend) & \\
\hline C7 & Infrarotspektroskopie & Chemische Verbindungen & \\
\hline C8 & $\begin{array}{l}\text { Atomabsorptionsspektroskopie } \\
\text { (AAS) }\end{array}$ & $\begin{array}{l}\text { Quantitativer Nachweis metallischer und } \\
\text { halbmetallischer Elemente }\end{array}$ & 7.7 \\
\hline C9 & $\begin{array}{l}\text { lonenchromatographie } \\
\text { LIBS (auch mobil seit 2018) }\end{array}$ & $\begin{array}{l}\text { Salzbestimmung, } \\
\text { Elementeverteilung }\end{array}$ & 7.8 \\
\hline C10 & Titration & Chloridbestimmung & 5.6 \\
\hline C11 & Thermogravimetrie & Stoffanalyse & 7.9 \\
\hline
\end{tabular}

Tabelle 7 Labortechnische Prüfverfahren für chemische Kenngrößen und physikalische Bestandteile 


\subsection{Röntgenfluoreszenz-Analyse}

In Verbindung mit der Rasterelektronen-Mikroskopie wird die chemische Zusammensetzung des Probenmaterials im mikroskopisch kleinen Bereich mit einem Röntgenspektrometer bestimmt.

\subsection{Infrarotspektroskopie}

Das stoffspezifische Strahlungsspektrum einer Probe gibt beim Durchgang von Infrarotstrahlung anhand der absorbierten Wellenlängen Aufschluss über chemische Verbindungen.

\subsection{Atomabsorptions-Spektroskopie (AAS)}

Bei der AAS wird die in die Gasphase überführte Baustoffprobe mit einer Spektrallampe durchleuchtet. Aus der Lichtschwächung berechnet sich die Elementkonzentration. Die AAS dient der Bestimmung der Bindemittelzusammensetzung und dem Nachweis von Natrium und Kalium bei einer AKR (Alkali-Kieselsäure-Reaktion).

Bei der Atomabsorptions-Spektrometrie wird das Licht einer speziellen Lichtquelle, die das Atomlinienspektrum des betreffenden Elements aussendet, durch eine die lnformation tragende Flamme geführt. Atome im Grundzustand schwächen dabei eine entsprechende Resonanzlinie der nachzuweisenden Atomart.

\subsection{Ionenchromatographie}

Die Ionenchromatographie bestimmt an einem wässrigen Auszug den Salzgehalt. In Abhängigkeit von der Wanderungsgeschwindigkeit von Ionen durch eine Trennsäule ergibt sich ein charakteristisches Chromatogramm, das den Nachweis über Schadstoffe ( $\mathrm{SO}^{4-}, \mathrm{Cl}^{-}$, $\mathrm{NO}^{3-}, \mathrm{NO}^{2-}$ ) erbringt.

\subsection{Thermogravimetrie}

Bei der Thermogravimetrie wird eine Probe, die nur wenige Gramm wiegt, kontinuierlich erwärmt. Dabei wird das Probengewicht als Funktion der Temperatur gemessen. Masseänderungen lassen sich der Verdampfungstemperatur bekannter Stoffkomponenten zuordnen. 


\section{Merkblätter und Beschreibungen von ZfPBau-Verfahren}

Wegen des geringen Normungsgrads und der wenigen Vorschriften spielt Fachliteratur bei der großen Bandbreite an ZfPBau-Verfahren eine wichtige Rolle.

\section{Bauphysik-Kalender 2004}

Der Bauphysik-Kalender ist eine Dokumentation des Stands der Technik für verschiedene ZfPBau-Verfahren und Prüfaufgaben.

\section{Merkblätter DGZfP}

Die Merkblätter der B-Reihe der Deutschen Gesellschaft für zerstörungsfreie Prüfung (DGZfP) verweisen auf weitere Verfahren.

\begin{tabular}{|c|c|}
\hline В 02 & $\begin{array}{l}\text { Zerstörungsfreie Betondeckungsmessung und Bewehrungsortung an Stahl- und } \\
\text { Spannbetonbauteilen (Ausgabe 2014) }\end{array}$ \\
\hline В 03 & $\begin{array}{l}\text { Elektrochemische Potentialmessungen zur Detektion von Bewehrungsstahlkorrosion } \\
\text { (Ausgabe 2014) }\end{array}$ \\
\hline B $03 \mathrm{E}$ & $\begin{array}{l}\text { Electrochemical Half-Cell Potential Measurements for the Detection of Reinforcement Corrosion } \\
\text { (Ausgabe 2014) }\end{array}$ \\
\hline B 04 & $\begin{array}{l}\text { Ultraschall-Impuls-Verfahren zur Zerstörungsfreien Prüfung mineralischer Baustoffe und } \\
\text { Bauteile (Ausgabe 2018) }\end{array}$ \\
\hline В 05 & $\begin{array}{l}\text { Aktive Thermographieverfahren zur Zerstörungsfreien Prüfung im Bauwesen } \\
\text { (Ausgabe 2013) }\end{array}$ \\
\hline B 06 & $\begin{array}{l}\text { Sichtprüfung und Endoskopie als optische Verfahren zur Zerstörungsfreien Prüfung im } \\
\text { Bauwesen (Ausgabe 1996) }\end{array}$ \\
\hline В 08 & $\begin{array}{l}\text { Seismische Baugrunderkundung } \\
\text { (Ausgabe 1996) }\end{array}$ \\
\hline B $08 \mathrm{E}$ & $\begin{array}{l}\text { Specification Seismic Methods for Site Characterization } \\
\text { (Ausgabe 2013) }\end{array}$ \\
\hline B 10 & $\begin{array}{l}\text { Radarverfahren zur Zerstörungsfreien Prüfung im Bauwesen } \\
\text { (Ausgabe 2008) }\end{array}$ \\
\hline B 11 & $\begin{array}{l}\text { Anwendung des Impakt-Echo-Verfahrens zur Zerstörungsfreien Prüfung von Betonbauteilen } \\
\text { (Ausgabe 2011) }\end{array}$ \\
\hline \multirow[t]{2}{*}{ B 12} & $\begin{array}{l}\text { Korrosionsmonitoring bei Stahl- und Spannbetonbauwerken } \\
\text { (Ausgabe 2017) }\end{array}$ \\
\hline & $\begin{array}{l}\text { Magnetische Verfahren zur Spannstahlbruchortung } \\
\text { (Positionspapier August 2017) }\end{array}$ \\
\hline
\end{tabular}

Tabelle 8 Merkblätter der B-Reihe der Deutschen Gesellschaft für zerstörungsfreie Prüfung 


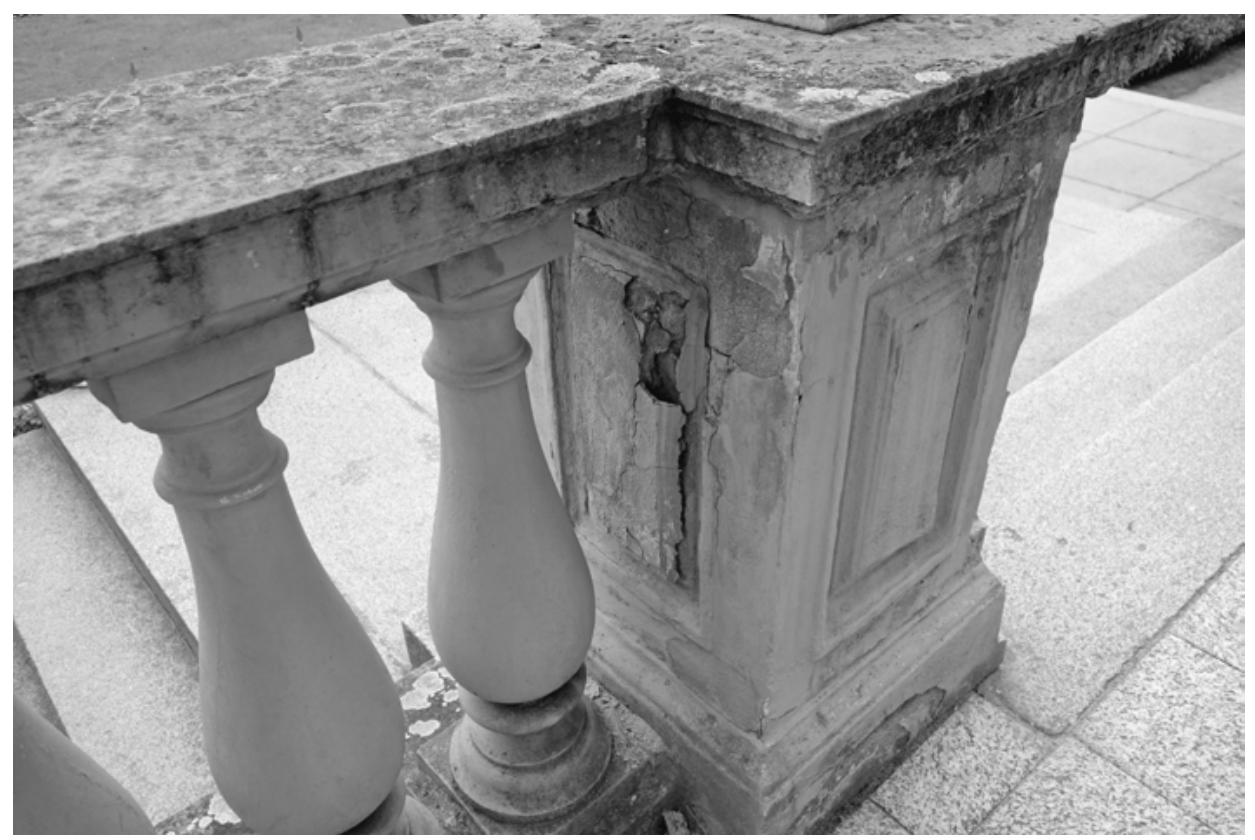

2 Fortgeschrittene Schäden in Branitz. Frühzeitige Detektionsverfahren für vorausschauende Maßnahmen im Rahmen eines Erhaltungsmanagements: (G1) (G3) (G4) (G5) (M1) (M3) (M7) (H2) (H3) (P2) (C9).

\section{Beispiele für die Anwendung einiger Verfahren}

Die aufgeführten Tabellen dienen als Checklisten. Die Spalten »Prüfverfahren« und »Untersuchungsziel« geben einen schnellen Überblick über Problemsituationen und Herangehensweisen. Jedes Verfahren erhält eine Kennung. G bedeutet geringer Aufwand, das Verfahren ist einfach, $M$ steht für mittelmäßig, mit erhöhtem technischem Aufwand, $H$ steht für hohen technischen Aufwand. P nennt physikalische und C chemisch/physikalische Laborverfahren. Erläuterungen und Literaturhinweise sind in der Tabellenspalte »Abschnitt « angegeben.

Die folgenden Beispiele dienen der Erläuterung der Anwendung der checklistenartigen Tabellen (Taf. VII und VIII, Abb. 2).

\section{Literaturverzeichnis}

Bauministerkonferenz - Konferenz der für den Städtebau, Bau- und Wohnungswesen zuständigen Senatoren der Länder (ARGEBAU) (2006): Hinweise für die Überprüfung der Standsicherheit von baulichen Anlagen durch den Eigentümer/Verfügungsberechtigten, Fassung September 2006. https://www.umwelt.niedersachsen.de/download/57359/Standsicherheit.pdf (22.10.2018). 
Bundesministerium für Verkehr, Bau und Stadtentwicklung (BMVBS) (Hg.) (2008): Richtlinie für die Überwachung der Verkehrssicherheit von baulichen Anlagen des Bundes, RÜV. https://www.fib-bund.de/Inhalt/ Richtlinien/RUV/richtlinie-fuer-die-ueberwachung-der-verkehrssicherheit.pdf (22.10.2018).

Bundesministerium für Verkehr, Bau und Stadtentwicklung (Hg.) (2017): (OSA) Leitfaden Objektbezogene Schadensanalyse. https://www.bast.de/BASt_2017/DE/Ingenieurbau/Publikationen/Regelwerke/Erhaltung/RI-ERH-ING-OSA-Leitfaden-Erhaltung.pdf?__blob=publicationFile\&v=3 (22.10.2018).

Hillemeier, Bernd; Taffe, Alexander (2012): Aktuelle Regelwerke der Bauwerksdiagnostik. In: BauphysikKalender: Gebäudediagnostik, 54-101.

DIN 1076: Ingenieurbauwerke im Zuge von Straßen und Wegen, Überwachung und Prüfung, Ausgabe 1999-11. Berlin: Beuth Verlag.

DAfStb-Richtlinie - Schutz und Instandsetzung von Betonbauteilen (RL SIB) - Teil 1: Allgemeine Regelungen und Planungsgrundsätze; Teil 2: Bauprodukte und Anwendung; Teil 3: Anforderungen an die Betriebe und Überwachung der Ausführung; Teil 4: Prüfverfahren, Ausgabe 2001-10. Berlin: Beuth Verlag.

VDI-Gesellschaft Bauen und Gebäudetechnik, Fachbereich Bautechnik (2010): VDI-Richtlinie 6200: Standsicherheit von Bauwerken - Regelmäßige Überprüfung, Ausgabe 2010-02.

\section{Bildnachweis}

1, 2 Bernd Hillemeier.

Taf. VII, VIII Bernd Hillemeier. 


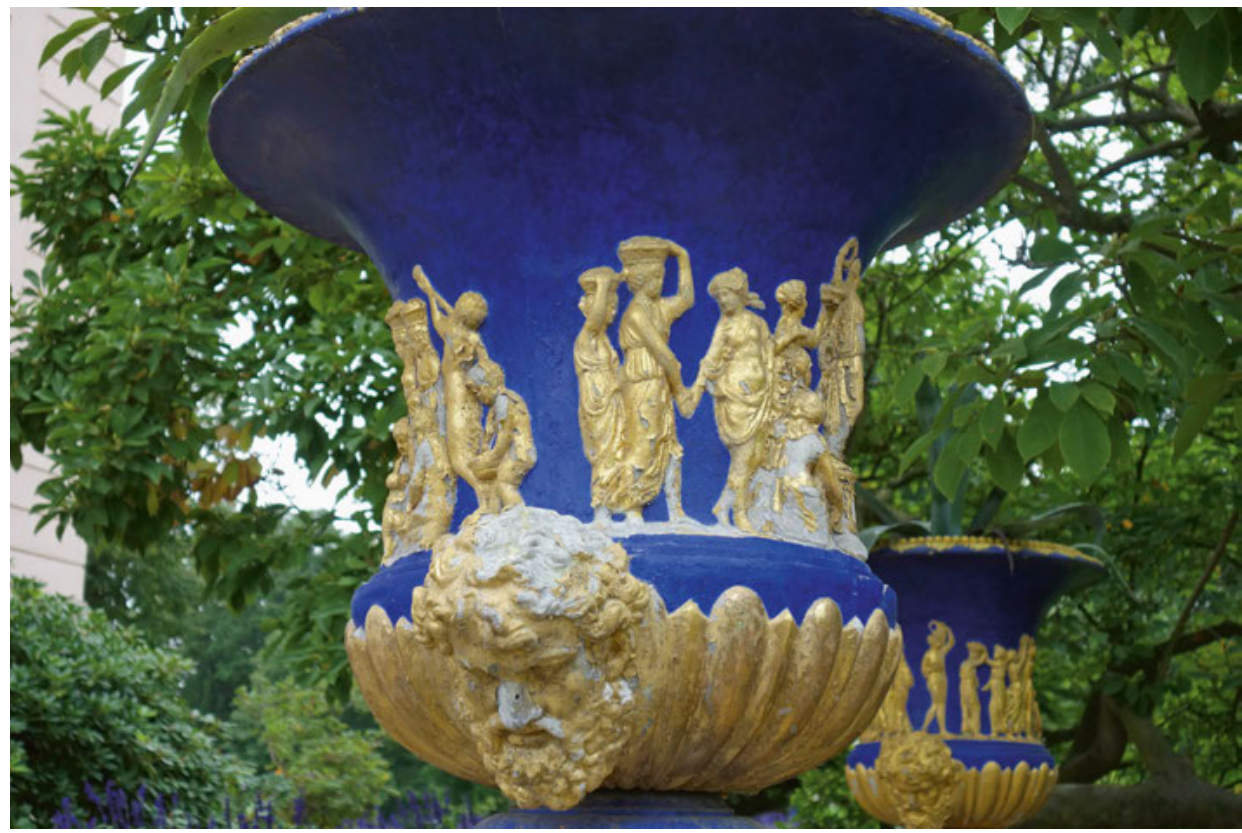

VII Branitz: Figuren in Gold. Vor der Restaurierung: (G1) (G3) (G4) (C9).

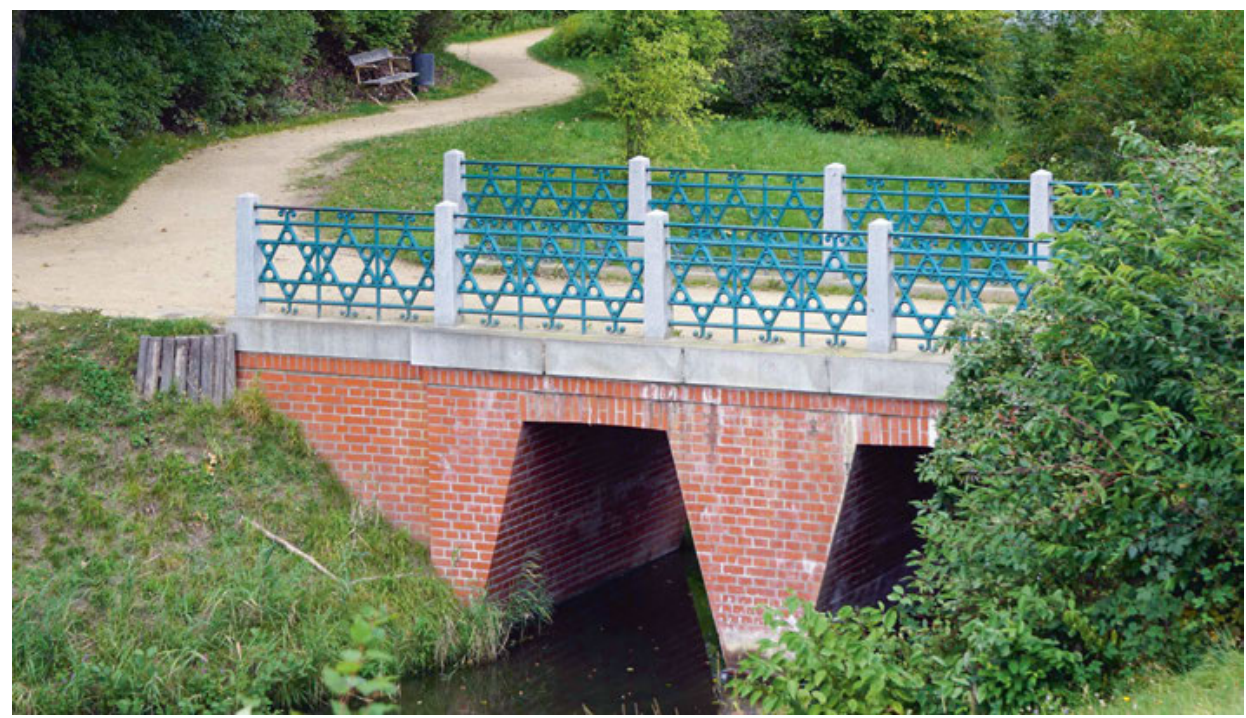

VIII Branitz: Mauerwerkbrücke: Verfärbungen nur durch undichte Fugen oder durch eine untüchtige Abdichtung? (G3) (G4) (G5) (M1) (H2). 